\title{
La deuxième ligne de l'inscription latine du duenos et l'interprétation du segment noisi (védique nédati, lituanien Niedà, lusitanien Langanidaeigui, Langanitaeco)
}

Vincent Martzloff - Barbora Machajdíková (Sorbonne University, Paris; Comenius University, Bratislava)

\section{The second line of the Latin Duenos inscription and the interpretation of the sequence noisi (Vedic nédati, Lithuanian Niedà, Lusitanian Langanidaeigui, Langanitaeco)}

\begin{abstract}
Considerable advances have already been made in elucidating the text of the Latin Duenos inscription (6th century BCE). Nevertheless, many difficulties remain, and the ongoing discussion has given rise to several misconceptions that ought to be cleared away. First of all, it is not a votive inscription, in spite of what is sometimes asserted. The fact that the Duenos vase may have belonged to a votive deposit does not imply that the vase and its inscription (incised before baking) were intended for religious purposes. The inscription is not of dedicatory nature. It has been suggested that uirco represents some kind of divinity (Proserpina, Athena, or Fortūna), but there is no positive evidence for such an interpretation. It needs to be emphasized that the words deiuos "gods" and mitat (also attested in the Tibur Pedestal inscription) do not allow the conclusion that the vase was initially intended to be a votive offering. The presence of deiuos (accusative plural) is to be explained by the mention of an oath (iouesat "iūrat"). The verb mitat ("gives" uel sim. rather than "sends") is better taken as referring to the aristocratic gift-exchange system. As for the function of the vessel, it has been interpreted as a receptacle for cosmetics. Alternatively, this small piece of elegant design may have been purely decorative and not intended for concrete use, like the Poggio Sommavilla fiaschetta (ST Um 2, around 600 BCE).

Several linguistic hypotheses have to be rejected. The alleged infinitives oitesiai (E. Sievers), oitesi (R. Godel), and oisi (E. Tichy) are excluded on morphological grounds. Since the long final /ī/ of amārì continues *ei, the sequence of six letters PACAR/ cannot be the ancestor of the Latin passive infinitive pācārī. The sequence UOIS cannot be the source of uīs "you want" because the [o] vocalism could not be accounted for. Since the Latin conjunction nisi is
\end{abstract}


a shortened form of nĭsi (which in turn may derive from nīsi < *neisei with a shortening similar to quăsi < quam sì), a comparison between NOISI and nisi is impossible. (It would of course be unjustified to correct the word to **NOISEI.) Moreover, the /o/ grade in the negation would be unexpected. The putative Umbrian parallel nosue (Tlg Vlb 54) that has been adduced as supporting the vocalism of the first part of noisi is not probative since nosue may be traced back to *nauswai <*n(e) au swai < *ne ayu(d) swai or *ne (h)ayu(d) swai, cf. the Latin ne-que haud (Plaut. Men. 371, Ter. Andr. 205; haud < * $\hat{g}^{h} / g^{h i} /$ e háey-u-d, cf. Vedic hí or gha) and the Greek oú $<$ *oyu < *hóy-u(d). Therefore, the interpretation of noisi as "nisi" has to be abandoned.

It is argued that the second line has to be segmented as follows: ast / ted / noisi / opet / oites / iai / paca / riuois. The analysis of oites [oite ${ }^{n} s s$ ] as a participle ancestor of the Latin ütēns (R. Thurneysen, R. Meringer, A. Götze) seems unassailable from a phonological point of view; regarding the omission of the nasal, cf. cosmis $<* k o n s m^{\circ}<* k o m-s m^{\circ}$ (not **ko-smi-s) and also Lat. qetios [kwentios] (cf. Quinctius, not Cottius), perhaps placiom (cf. Plancius), Etr. araz (Rome, S. Omobono, 580-540, cf. arant, Arruns). As expected, oites governs the ablative case (riuois). The fossilized adverbial locative iai may be equated with the first part of the Umbrian iepi $<* i y a \bar{a}-k^{w}$ id (containing the same ending as the Oscan svai), hence a complex conditional system nei..., ast..., iai... "if... not..., and/but..., then..." (asted < ast + tēd similar to astu < ast $+t u \bar{i}$ in the protocols of the Arval Brothers).

The verb opet (<*h,op-éye-ti, cf. optāre) is construed with the ablative (ted... opet "(if) she wants from you"). Although it may at first seem counter-intuitive, opet takes a genitive object according to a construction represented in Early Latin with verbs of desiring (studeat tuĩ, domi cupiō). Since the girl (still a uirgō) has to be satisfied with the discharge of some fluids (riuois, a classic case of a double entendre), it seems fruitful to turn our attention to the possibility that the word noisi itself refers to a liquid. It is tempting to establish a connection between noisi and the root of the Vedic nédati "to overflow" (cf. the Khotanese gganih- "to moisten"), which is well attested in European hydronymy (Nida in Poland, Niedà in Lithuania, La Nied in Lorraine, Nidd in Yorkshire, cf. also the Lusitanian Langa-nida-eigui).

Regular sound laws suggest for noisi the proto-form *noid-to-s > /noissos/ (as tussis < *tudti-s, cf. tundō), with a genitive /noissī/ spelled NOISI. The formation *noid-to-s is structurally comparable to * $\hat{g}^{h}$ or-to-s (Latin hortus, Oscan húrz, Greek xóptos, Old Irish gort). A *C(C)oC(C)to-s formation is precisely attested for the semantically related root *pleu- (the Greek $\pi$ तoũtoc: "flood" > "large quantity" > "wealth"). The use of the partitive genitive is further facilitated when such a meaning is assumed for noisi (cf. impleto aquae purae). The Duenos inscription thus contains a facetious advertisement with erotic overtones in which riuois (separated from its governing participle oites to create a punchline) is co-referential with noisi.

\section{Keywords}

case syntax, genitive, Italic languages, hydronymy, Lusitanian, Old Latin, Umbrian. 
L'inscription latine du duenos, datée de la première moitié (deuxième quart?) du VI ${ }^{\mathrm{e}}$ siècle avant notre ère, a été gravée avant cuisson sur un vase constitué de trois petits récipients réunis de façon à former un triangle équilatéral. ${ }^{1}$ Le texte, réparti en trois séquences (improprement appelées "lignes"), est complet et entièrement lisible. La lecture a fait l'objet de minutieuses discussions de la part de Hempl (1902), Gordon (1975) et Prosdocimi (1979):

\section{[1] ioue/satdeiuosqoimedmitatneitedendocosmisuircosied \\ [2] astednoisiopetoitesiaipacariuois \\ [3] duenosmedfecedenmanomeinomduenoinemedmalostatod.}

La présence de plusieurs traces de corrections par le graveur (en particulier l'insertion de la lettre $U$ dans duenoi) démontre que le texte est sûr: il ne doit donc pas faire l'objet de conjectures de la part des chercheurs qui tentent d'en donner une interprétation.

Depuis sa publication par Dressel $(1880)^{2}$ le texte a donné lieu à de nombreuses tentatives herméneutiques, dont les plus anciennes ont été résumées par Goldmann (1926) et St. Marin (1950). Un état de la recherche ultérieur est donné par Eichner (1988-1990). L'interprétation de la deuxième ligne a suscité d'intenses controverses, dont les derniers développements ont été présentés et commentés par Martzloff (2015). Dans son étude, ce dernier auteur a proposé une analyse nouvelle pour le segment écrit IAI (rapproché de l'adverbe ombrien ie-pi), mais il a été contraint de laisser sans explication la séquence de cinq lettres NOISI. Nos efforts porteront ici précisément sur l'analyse de NOISI.

\section{Présentation du vase de Dressel et considérations méthodologiques}

Nous commençons par souligner un point essentiel pour l'interprétation: l'inscription de Dressel $n$ 'est pas une inscription votive, contrairement à ce que plusieurs chercheurs ont supposé (explicitement ou non). Nous mettons en garde contre le paralogisme suivant: "le vase provient d'un dépôt votif, donc le vase a une destination votive, donc l'inscription, gravée avant cuisson, fait référence à la finalité votive du vase”. Ce raisonnement simpliste a donné lieu à des contresens majeurs. Ainsi, nous rejetons fermement l'affirmation de Coarelli (1988: p. 289) d'après laquelle "il vaso non è un dono, ma un

1 Nous parlons de l'inscription "du" duenos (avec article) et non de l'inscription "de" Duenos, car il est désormais admis que duenos et duenoi ne sont pas des noms propres, mais des adjectifs substantivés, représentant des formes anciennes du paradigme de bonus "bon", dont l'assemblage constitue un polyptote. Autre conception chez Dressel (1880: p. 183, note 2) et Adamik (2010: pp. 21, 24). [Notre travail a été réalisé dans le cadre du projet VEGA 1/0812/18 "Latinská slabika v diachronickom a typologickom kontexte". Pour leurs précieux commentaires sur des points ciblés, nous remercions Heiner Eichner, Jean Hadas-Lebel, Georges-Jean Pinault, Murad Suleymanov, Michael Weiss et deux reviewers anonymes. Les thèses exprimées ici n'engagent que nous.]

2 Les circonstances de la découverte du vase sont retracées par Pinza (1905: col. 259-260, 643-649), qui traite également les aspects matériels de l'objet. 
ex-voto, o comunque un oggetto in qualche modo sacro, deposto in un santuario". On ne suivra pas les conclusions de Coarelli (1988: p. 290) qui prétend être en mesure "di riconoscere nel nostro vaso un kernos di un tipo particolare, contenente primizie o piuttosto altre sostanze rituali, e quindi destinato fin dall'origine [nous soulignons] ad essere offerto in un santuario."

Certaines thèses de Coarelli sont fondées sur des présupposés linguistiques manifestement erronés. Ainsi, Coarelli (1988: p. 287), trop influencé par Dumézil (1969: p. 19; 1979: p. 97), a imaginé que la deuxième ligne renfermait un théonyme apparenté à Tütêla. Il est pourtant rigoureusement impossible que le segment écrit TOIT soit apparenté à tūtēla, dont la racine ne contenait pas de $/ \mathrm{i} / .^{3}$ Enfin, la suggestion de Coarelli (1988: p. 293) d'après laquelle la uirco serait une "porné legata al santuario" suscite les plus grands doutes.

Contrairement à ce qu'affirme Prosdocimi (2010: pp. 384-385), un nom propre **Toitesiai n'existe probablement pas. Et il ne se trouve pas l'ombre d'un argument textuel en faveur de l'idée que uirco désigne la déesse Fortūna, comme le voudrait Prosdocimi. Ce mot ne renvoie pas non plus à Proserpine, ni à Athéna, comme l'a proposé Fraser (1932: p. 226).

Selon nous, dans l'esprit des artisans (potier, graveur) qui en ont assuré la fabrication, le vase n'était pas destiné à être confiné dans un sanctuaire. Au contraire, des indices internes à l'inscription montrent que le vase avait pour vocation de circuler d'un propriétaire à un autre. L'absence de nom propre (duenos et duenoi n'étant pas des anthroponymes) facilitait la circulation de l'objet, car les identités du donateur et du destinataire restaient ouvertes. En outre, plusieurs éléments textuels (verbe mitat "(il) offre", construction de feced avec le datif du destinataire) constituent une mise en abyme de cette circulation de l'objet entre les gens de bien. Nous sommes donc pleinement d'accord avec Colonna (1979: p. 168): “Oggetti letterati di questo genere, il cui pregio stava spesso in primo luogo nell'iscrizione, suscettibile di arricchimenti ed elaborazioni di carattere letterario, erano destinati a circolare prima di finire deposti in una tomba o in una stipe votiva, come appunto è accaduto al vaso di Duenos."

En conséquence, c'est secondairement que le vase de Dressel a dû se retrouver dans un dépôt votif, auquel il n'était pas destiné au moment de sa fabrication. "Destinato a circolare entro la cerchia ristretta di un pubblico litterato, di alto ceto, fu utilizzato secondariamente come offerta votiva”, écrit excellement Cristofani (1990: pp. 20-21). Eichner (1988-1990: pp. 207, 220, note 20) parvient à la même conclusion ("erst in sekundärer Verwendung einem Heiligtum dediziert").

Afin de décrire et de catégoriser le vase de Dressel, plusieurs auteurs ont employé le terme proprement grec de kernos: Benndorf ${ }^{4}$ (1900: p. 9), Fraser (1932), St. Marin (1950:

3 La racine de tütēla est *teuH- (cf. tueor). Voir Rix (1985: p. 212) et Rix \& Kümmel (2001: p. 639). Il est donc impossible que cette racine conduise à une séquence écrite >toi<. Donc tūtēla ne peut pas avoir présenté la même évolution phonétique *oi $>\bar{u}$ que le numéral ūnus (provenant de *oinos, cf. gotique ains) ou lüdus (graphies archaïques: loidos, loedos) ou mürus (graphie moiros).

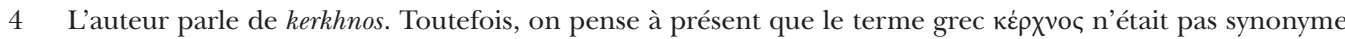
de кépvoc. Voir Bakalakis (1991: p. 110, note 20), avec bibliographie. 
p. 458). Colonna (1979: p. 165) parle de "minuscolo kernos d'impasto". Certes, mais la ressemblance très vague ${ }^{5}$ avec les kernoi n'autorise pas à conclure que le vase avait nécessairement une fonction religieuse ou votive. Plusieurs chercheurs ont pensé que le vase constituait un présent amoureux par lequel le soupirant (interpellé par le pronom personnel ted) devait pouvoir gagner les bonnes grâces de la jouvencelle (uirco). Le vase avait peut-être alors pour rôle de contenir des onguents, des cosmétiques (sous forme liquide ou sous forme de pâte, comme nos palettes de maquillage) ou des échantillons de nourriture (condiments de choix, épices, aromates, confiseries?).

Toutefois, une autre hypothèse est également possible: par ses dimensions très réduites, le petit vase du Quirinal est à mettre sur le même plan que la gourde miniature inscrite de Poggio Sommavilla (datée des environs de 600 avant notre ère), qui, selon nous, est une imitation des gourdes de pèlerin (pilgrim flasks, fiasche da pellegrino, Feldflaschen) en bronze étudiées par Marzoli (1989). ${ }^{6}$ Nous voulons suggérer que ces deux objets, la fiaschetta sabine, dont les arêtes imitent un prototype métallique, et le vase de Dressel, qui rappelle certes d'autres vases triples ${ }^{7}$ (comme les exemplaires de Valvisciolo, de Vignanello, des Monts Albains et surtout de Blera), étaient des bibelots (gingilli) purement décoratifs ou symboliques, sans réelle vocation utilitaire, même si les inscriptions évoquent une mise en scène fictive de leur utilisation.

Enfin, nous rappelons que la gourde sabine de Poggio Sommavilla, qui représente un objet typiquement masculin, a été retrouvée dans une tombe de femme, comme le précise Salskov Roberts (1974: p. 102). Cela démontre que cet objet portatif était susceptible de circuler et de changer de propriétaire, dans le cadre d'un échange ou d'un don entre particuliers. Nous pensons précisément que le vase du duenos lui aussi était destiné à circuler entre différents propriétaires: fabricant, premier donateur (le duenos qui l'offre au duenoi), second donateur (l'autre homme de bien qui le destine à la donzelle) et enfin la uirco elle-même.

En ce qui concerne les préceptes méthodologiques que nous pensons devoir suivre dans notre enquête, il convient de distinguer soigneusement la morphologie et le lexique. D’un côté, dans le domaine de la morphologie, nous refuserons toutes les hypothèses faisant intervenir des formations (suffixes ou types flexionnels) étrangères à la langue latine, telle que nous la connaissons. D’un autre côté, dans le domaine du lexique, il sera inévitable de faire intervenir des radicaux extérieurs au corpus du latin littéraire.

5 Contrairement à une pratique admise par beaucoup de chercheurs, il ne suffit pas que plusieurs petits vases soient assemblés pour qu'on puisse légitimement parler de "kernos". Il existait une tradition italique de vases multiples, de formes (et de fonctions) d'ailleurs très diverses. Pour s'en convaincre, on consultera les superbes illustrations données par Montelius (1904: planche 280) et Hencken (1968: I, p. 413).

6 Inscription ST Um 2, les sigles des documents sabelliques étant pris au recueil de Rix (2002). Sur ce texte, voir Machajdíková \& Martzloff (2016: p. 95, note 148). Contrairement à une idée souvent répétée, il n'existe probablement pas de lien génétique entre la gourde de Poggio Sommavilla et les bouteilles de Nouvel An égyptiennes (New Year's bottles, fiasche egizie di Capodanno, ägyptische Neujahrflaschen).

7 Bibliographie dans Martzloff (2015: p. 76). Le vase de Blera est représenté sur la planche XLII associée à l'article de Colonna (1979). Nous ajoutons une pièce provenant d'Auvernier (lac de Neuchâtel), publiée par Munro (1890: pp. 43-44, figure 9.23), sur laquelle Pinza (1905: col. 248) et Ducati (1938-1939: p. 14) ont attiré l'attention. 
La légitimité de cette approche est illustrée par l'interprétation de tatod donnée par Rix (1985), qui a expliqué le dernier mot du texte tatod comme l'impératif "futur" d'un verbe contenant la racine *teh ${ }_{2^{-}}$"voler, dérober, subtiliser, chiper" attestée dans différentes langues: hittite tāyezzi "il vole", vieil irlandais táid "voleur", vieux slave tatı̆ "voleur" et tajo, tajiti "voler" (slovaque tajit" "dissimuler"), védique stāyat (adv., AV) "secrètement",

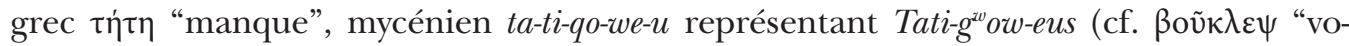
leur de vaches"). ${ }^{8}$ L'évocation d'un larcin figure dans l'imprécation hòc $\delta$ ' ăv $\mu \varepsilon \kappa \lambda \varepsilon \dot{\varepsilon} \varphi \sigma \varepsilon$ ।

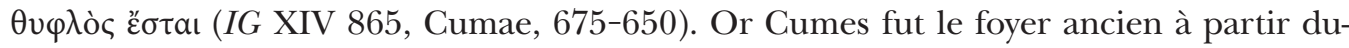
quel l'hellénisme a rayonné en Italie centrale. Citons aussi noli me tollere Helueiti sum (CIL $\mathrm{I}^{2}$ 2376). L'emploi solennel de l'impératif "futur" dans ne med malos tatod "qu'un vaurien ne me dérobe pas" semble être un pastiche du style juridique. ${ }^{9}$

Cette analyse de tatod a été rejetée par Stefanelli (2012: p. 226) sous le prétexte que la racine n'est pas attestée en latin. Mais l'identification de la même racine en sabellique dans atahus (ST VM 2) démontre qu'elle était présente dans le lexique du proto-italique, ce qui suffit à invalider les doutes exprimés par Stefanelli. (Nous ajoutons que la prétendue construction transitive de statod analysé comme l'impératif "futur" de l'intransitif stāre est impossible.) Il faut donc tenir compte de la déperdition de mots du lexique entre le $\mathrm{VI}^{\mathrm{e}}$ siècle et l'époque où le latin commence à être attesté massivement (avec le théâtre plautinien). Ce phénomène est mis en évidence aussi par la disparition du verbe mitat, qui est attesté plusieurs fois en latin archaïque (vase de Dressel, base de Tibur).

\section{2. État de nos connaissances}

Dans une étude brève et lumineuse, Grienberger (1900) a identifié iouesat comme le précurseur de iürat (par rhotacisme), en rapprochant iouestod (Cippe du Forum). Le même auteur a analysé deiuos comme un accusatif pluriel dépendant de iouesat: "il jure par les dieux". C'est un emploi constatif (et non performatif) du verbe de serment, à la troisième personne, comme l'observe Poccetti (2019: p. 516). L'osque a un verbe délocutif de serment deiuatud.

Le sujet de iouesat est la relative introduite par qoi (nominatif masculin singulier). Ce qoi est le sujet de mitat (indicatif présent). Même si le verbe mitat est attesté également sur la base de Tibur, Poccetti (2009: p. 60) a raison d'écrire que l'expression qoi med mitat du vase de Dressel "sicuramente non ha alcun riferimento votivo".

Il n'est pas sûr que mitat soit apparenté au verbe latin mittere "envoyer". ${ }^{10}$ Malgré Rix (1999: p. 530, note 38), il n’est pas nécessaire de rejeter le témoignage de Paul Diacre qui cite une forme cosmittere (Paul. Fest. 59, 5 L) avec un /s/. D'après Vine (1999), il

8 Selon une intéressante proposition de Lipp (2009: p. 321, note 16), on peut mettre en relation la racine attestée dans tatod (cf. atahus et peut-être marrucin $t a[-] a, S T$ MV 1) avec la racine élargie $* t h h_{2} k$ - du verbe latin tacēre "se taire" (gotique pahan, vieux haut-allemand dagēn, arménien $t^{\star} a k^{\star} c^{\star} i m$ ).

9 Sur les fonctions de l'impératif en *-tōd dans les langues italiques, voir Poccetti (2018).

10 Nous attirons toutefois l'attention sur une dédicace béotienne à Apollon Ptoien (VI siècle) qui emploie

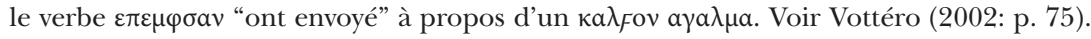


est préférable de rattacher mitat à la racine attestée dans mūnus "office; don, présent, faveur" < *(h $\left.h_{2}\right)$ moi-n-os et dans meāre <*mey- $\bar{a}$ - (avec amuïssement régulier $\mathrm{du} / \mathrm{y} /$ intervocalique) "passer, circuler" (c'est-à-dire "changer de lieu”). Le sens premier de mitat devait être "donner en échange”. Les formes sud-picéniennes meitims (ST Sp TE 5) et meitimúm (ST Sp AP 2) semblent être apparentées à mitat et rappellent le substantif gotique maipms "don”. Donc qoi med mitat signifie "celui qui me donne, celui qui m’offre, celui qui me remet” (plutôt que "celui qui m’envoie”).

Dans la subordonnée introduite par la conjonction nei "si... ne... pas", le substantif uirco (uirgō) "vierge, jouvencelle" est le sujet du subjonctif sied (latin archaïque siet, plus tard remanié en sit). L'adjectif prédicat est cosmis, ancêtre de cōmis "affable". L'adjectif cosmis est construit avec la postposition endo, dont le régime est ted: "aimable envers toi".

Les connotations érotiques de cosmis ont été soulignées par Pinault (2013: pp. 34-35). On a voulu voir en cosmis un composé contenant la racine *smei- de smáyate "sourire" dans le second membre, mais on attendrait *kom-smi-t-s (avec élargissement en dentale). Pinault opte finalement pour un composé possessif en ${ }^{*}{ }^{\circ}$ smid-s $>{ }^{*}{ }^{\circ}$ smiss (racine $*$ smeidde grec $\left.\mu \varepsilon ı \delta เ a ́ \omega, \varphi \iota \lambda_{0}-\mu \mu \varepsilon ı \delta \eta ́ \varsigma\right)$, remanié en thème en /i/, peut-être d'après iuuenis. [Ceux qui accepteraient de poser une racine à laryngale finale malgré le participe smita- (cf. moyen anglais smìlin) pourraient comparer le traitement de cosmis $\ll *{ }^{\circ}$ smiH-s à celui de iūgis "qui coule toujours", remaniement de $* h_{2} i(e) u-g^{z v} i h_{3}-s$, absorbé dans les thèmes en /i/. Mais les formes germaniques sont susceptibles d'autres interprétations.]

L'analyse correcte de asted comme ast + ted (avec une notation des deux / t/ consécutifs par une lettre $T$ unique) a été proposée par Bréal (1882: p. 157). (Nous rappelons que Michel Bréal est également le premier à avoir lu correctement DUENOI et MALO(S).) Cette analyse est confirmée par l'existence d'une forme écrite ASTU (représentant ast $t \bar{u}$ ) qui apparaît à de nombreuses reprises dans les protocoles de la Confrérie Arvale, à l'intérieur de la formule stéréotypée astu ea ita faxis (ou facxis) "et si toi tu réalises cela de cette manière" (parfois attestée sous forme fragmentaire). Les références sont: $\mathrm{n}^{\circ} 12 \mathrm{a}$ : 1 (Scheid 1998: pp. 28, 32), n²2: 13, 20, 27 (Scheid 1998: pp. 52, 53), n59 I: 8, 11, 15, 19 (Scheid 1998: pp. 164, 166), n62a: 35, 39, 46, 50, 57, 60, 63, 66, 69 (Scheid 1998: pp. 178, 179, 181, 182), n`86: 5 (Scheid 1998: p. 249). En revanche, l'analyse de asted comme subjonctif de adstāre n'est pas vraisemblable, car on attendrait plutôt **adsted à époque ancienne, comme le suggèrent les formes sud-picéniennes adstaeoms (ST Sp CH 1a) et adstaiuh (ST Sp AP 2).

La barre verticale dans ioue/satdeiuos n'a pas reçu d'explication satisfaisante. La bibliographie est rassemblée dans Martzloff (2015: pp. 78-79). Nous proposons l'analyse suivante: les deux premiers mots du texte, qui sont étroitement associés du point de vue sémantique, forment une micro-séquence de 13 lettres, au centre de laquelle les lecteurs antiques étaient incités à découvrir un segment de 5 lettres $S A T D E$, qui était délimité à droite par la barre verticale: ioue-SATDE-iuos. Ce segment SATDE correspond exactement, par une double permutation calculée des lettres ( $S$ et $A, D$ et $E$ ), à la séquence graphique $A S T E D$ formée par les deux premiers mots de la deuxième ligne (ast têd).

La barre avait donc pour rôle de mettre en évidence un effet de seuil, consistant en une correspondance entre les débuts des deux premières lignes de l'inscription. La particularité 
de cet effet de seuil est qu'il est décalé au sein de la première ligne, puisqu'il met en jeu un segment textuel qui commence après la quatrième lettre. C'est précisément ce décalage que la barre verticale avait pour fonction d'indiquer:

ioue / SATDEiuosqoimedmitat ... ASTEDnoisiopet ...

En plus de cet effet de seuil était également présent un effet de clôture, puisque les 4 lettres suivantes IUOS de la micro-séquence de 13 lettres (après $S A T D E$ ) se retrouvent à l'extrême fin de la deuxième ligne (UOIS). Enfin, les deux premiers mots du texte sont encadrés par la même séquence permutée de 5 lettres: IOUES-atd-EIUOS. Il s'agit d'une mise en scène ludique de l'écriture, mettant en jeu un procédé que nous appelons stéganographie.

Sur le plan phonétique, les 4 premières consonnes de la deuxième ligne sont les mêmes que les 4 dernières consonnes de la troisième ligne (sans permutation): $a \boldsymbol{S T} \boldsymbol{T} \boldsymbol{T} \boldsymbol{D} \ldots$ (malo)S $\boldsymbol{T} a \boldsymbol{T} o \boldsymbol{D}$. Un phénomène voisin s'observe sur l'inscription sud-picénienne de Bellante (ST Sp TE 2), à ceci près qu'à Bellante, cinq consonnes sont concernées et qu'on y observe une permutation: PoSTiN V(iam) ... (vepse)S VePeTeN.

La suite de la deuxième ligne est controversée. Il nous semble utile d'énumérer et de rejeter explicitement plusieurs analyses qui sont manifestement erronées. Le point essentiel est que la séquence de six lettres PACARI ne peut pas (en dépit de trompeuses apparences) être interprétée comme l'équivalent de l'infinitif passif pācārì, car le /ì) long final de l'infinitif passif remontait à une diphtongue /ei/. Or les diphtongues sont conservées dans la langue du vase de Dressel, comme le démontrent deiuos (deōs), nei $(n \bar{\imath})$, qoi (quī), duenoi (bonō) et probablement aussi oites (ūtêns) et riuois (rīū̄s). L'existence d'un infinitif pacari, encore admise par Stefanelli (2012: pp. 211, 219), est donc illusoire. ${ }^{11}$ En outre, l'analyse de la séquence UOIS comme précurseur de uĩs "tu veux" est exclue, car le vocalisme [o] serait incompréhensible, en dépit des allégations de Stefanelli (2012: p. 215).

Un infinitif oitesiai, postulé par Sievers (1930: p. 54) et Harđarson (2011: pp. 159160), est difficile, car il aurait conduit à **ūterier, alors que la forme latine correcte est ütier (Plaut. Cas. 220, Cist. 24, Truc. 734). L'infinitif actif oitesi proposé par Godel (1962: p. 104) est douteux, car ütor est un verbe déponent. L'idée d'un infinitif oisi et d'un impératif oit (à la fois athématiques et actifs) proposée par Tichy (2004: p. 198) est audacieuse et peu réaliste. Nous renvoyons aux discussions critiques de Martzloff (2015: p. 96; 2018: p. 235).

La séquence NOISI a souvent été interprétée comme l'équivalent de nisi "si... ne... pas”. Déjà Dressel (1880: p. 180) avait comparé NOISI à la conjonction ombrienne nosue (TIg VIb 54) "nisi”. Plusieurs savants, comme Eichner (1988-1990: pp. 213, 234) et Harđarson (2011: p. 157), ont accordé un grand poids à cette forme nosue, car elle semble confirmer la présence d'un vocalisme /o/ dans la négation. En réalité, le vocalisme de nosue est susceptible de différentes explications.

La comparaison des vocalismes notés $O$ dans nosue et dans sopir (TIg VIb 54) ne permet aucune conclusion: sopir n'est pas issu de $*^{*}$ soi- $k^{w}$ is (qui serait une reconstruction

11 Nous signalons une tentative pour échapper à cette contradiction chez Tiffou (1980: pp. 227-228). 


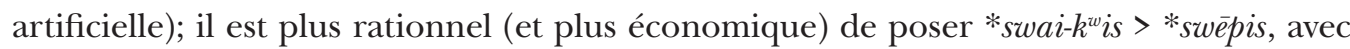
une forte érosion phonétique (due au statut de mot grammatical) en *swěpis [cf. sĭ-qui$d e m]>*_{s}(w)$ opis (avec une altération du timbre vocalique dans les registres progressifs de l'ombrien récent). Le $O$ de nosue n'a probablement aucun lien avec le $O$ de sopir.

Nous proposons d'expliquer le nō- de nosue comme le reflet de *nau<*n(e) au<*ne ayud ou *ne hayud (cf. lat. neque + haud dans Plaut. Men. 371, Ter. Andr. 205), en supposant que haud s'analyse comme $h$-aud, reflet de l'univerbation de *h(e/i) ayud représentant * $g^{h} e h_{2} e ́ y-u-d$ ou $* \hat{g}^{h} i h_{2} e ́ y-u-d$ (où la particule initiale serait apparentée à védique (ná) gha, ou (na)hi, grec oủ-xí), tandis que la négation grecque oủ reflète $* h_{2} o ́ y-u(-d)$ désaccentué. ${ }^{12}$

L'interprétation de NOISI comme ancêtre de "nisi" est également rendue caduque par le fait que nŭš̆ (avec deux voyelles brèves) résulte de l'abrégement iambique de nŭsì (avec une voyelle longue dans la deuxième syllabe). Deux analyses ultérieures sont possibles: ou bien nŭsī remonte à *nesei, ou bien nŭsì remonte à nīsì (<*neisei) avec deux longues, dont la première a été abrégée comme dans sĭquidem, quŏque et surtout quăsi issu de quam sī (érosion phonétique dans un mot grammatical désaccentué), selon Brugmann (1909: p. 83) et Harđarson (2011: p. 158). Dans les deux cas, on aboutit à des reconstructions qui sont incompatibles avec NOISI. Il est illégitime de supposer que NOISI est une simple erreur pour *NOISEI, comme le propose Dunkel (2014: p. 740). Il n'existe donc aucun moyen raisonnable de sauver l'interprétation de NOISI comme "nisi". Cette identification probablement chimérique doit être résolument abandonnée.

\section{La séquence oites iai paca riuois}

Une segmentation paca / riuois (au lieu de pacari / uois) a été proposée par Steinbauer (1989: p. 35). Cette intéressante idée a été portée à la connaissance de la communauté scientifique par Eichner (1988-1990: pp. 214, 236). La division de Steinbauer a entièrement renouvelé l'étude de l'inscription du duenos. L'avantage de cette segmentation est qu'elle permet d'extraire de la scriptio continua des formes irréprochables du point de vue de la grammaire: un impératif $p_{a c a^{13}}$ et un ablatif (ou datif) pluriel riuois du lexème latin rìuus "ruisseau". L'inconvénient est qu'il semble difficile d'imaginer un contexte dans lequel l'allusion à des "ruisseaux" (ou à des "écoulements") soit compréhensible. Mais nous verrons que notre analyse de noisi permettra de mieux comprendre la présence de ce mot riuois.

[Une segmentation différente serait, en théorie, possible: pacar / riuois (avec la notation des deux /r/ par une seule lettre $R$, comme dans ASTED notant ast têd). Ce pacar

12 La terminaison *-u-d représenterait un instrumental archaïque de thème en / $\mathrm{u} /$ (hittite ge-nu-ut, ga-nu-ut), même si haut se rencontre à côté de haud. L'évolution *[au]> [ō] est régulière en ombrien: toru <*taurōns ou *taurons (cf. latin taurus). Voir Dunkel (2014: pp. 353, 540), Garnier (2017: pp. 243-253), Machajdíková \& Martzloff (2016: p. 87), Martzloff (2019: pp. 114, 120).

13 Le thème verbal pācā-est-il à mettre en relation avec l'ablatif ombrien paca (TIg VIa 20)? Le paca ombrien est-il un dérivé inverse d'un tel thème verbal? Si oui, le verbe serait ancien. En revanche, le verbe pācäre du latin classique, en raison de sa sémantique, a été re-dérivé de $p \bar{a} x$. 
serait la première personne du singulier du subjonctif présent du verbe thématique déponent d'où le verbe déponent pacīscor a été dérivé: ainsi, pacar aurait la même terminaison que le subjonctif ütar de ütor. Nous soulignons que ce verbe *pacor "faire un pacte, conclure un arrangement" pourrait être différent (en dehors de la voix) des formes du type pacunt, qui peuvent représenter pagunt.]

Plusieurs savants ont essayé de retrouver dans la séquence de trois lettres OIT le radical du verbe latin ūtor (la diphtongue /oi/ est préservée par exemple dans oitile, CIL ${ }^{2}$ 586). Si on accepte cette piste de recherche, il faut découvrir dans le texte une forme qui soit le précurseur d'un élément du paradigme de ütor réellement attesté dans le corpus du latin littéraire. Puisque ūtor est un verbe déponent, il possède un participe présent de forme active: ütêns. Or Thurneysen (1899: pp. 198, 212) et d'autres auteurs, comme Meringer (1907: p. 307) et Martzloff (2015: p. 97), ont supposé que OITES représentait la notation de l'ancêtre de ce participe ütêns.

La graphie OITES correspondrait à [oite $\left.{ }^{\mathrm{n}} \mathrm{ss}\right]$ issu de *oit-ent-s, avec une consonne nasale affaiblie et non notée (peut-être avec nasalisation de la voyelle) devant /ss/double. La consonne nasale a ensuite été restaurée dans ütêns, d'après les formes du paradigme ayant /nt/, tandis que OITES présente le traitement phonétique spontané devant sifflante double. On fera contraster ENDO.

Cette interprétation implique qu'on renonce à la conception trop souvent admise d'après laquelle une nasale anté-consonantique devait être systématiquement notée dans le latin des $\mathrm{VI}^{\mathrm{e}}-\mathrm{V}^{\mathrm{e}}$ siècles. ${ }^{14}$ Une telle conception est manifestement non fondée. Plusieurs considérations (dont certaines au moins sont significatives) doivent être prises en compte ici. Il conviendrait de distinguer avec soin (quand cela est possible) les cas d'amuïssement phonétique complet et les cas d'affaiblissement avec omission graphique.

- (1) À titre de parallèle, Götze (1923: p. 148) a observé que le mot cosmis devait refléter *konsmis (avec nasale) et a déjà précisément comparé l'affaiblissement de la nasale devant /sm/ avec OITES. En effet, à date ancienne, la variante ko- (sans nasale) ne se rencontrait normalement que devant $w^{15}$ et secondairement devant voyelle (et $h$ ) dans des composés productifs, à la différence de comes, comedō (résiduels). Selon nous, la reconstruction de $* k o-s m^{\circ}$ sans nasale est erronée: il faut nécessairement poser *kom-sm ${ }^{\circ}$ $>$ * $_{\text {konsm }}{ }^{\circ}$ et supposer un affaiblissement phonétique de la consonne nasale devant $/ \mathrm{s}(\mathrm{m}) /$, qui s'est produit dans la préhistoire récente du latin.

- (2) Dans l'accusatif pluriel deiuos, la réduction du groupe final */ns/ est "post-Proto-Italic", comme le souligne Weiss (2009: p. 171): cf. ombrien turuf. Mais les chronologies de l'amuïssement de /n/ dans /ns/ et dans /nss/ issu de *nts ne sont pas nécessai-

14 On trouve par exemple censores (CIL $\left.\mathrm{I}^{2} 2439\right)$, atriensis (CIL $\left.\mathrm{I}^{2} 1447\right)$, mais cosentiont (CIL $\mathrm{I}^{2}$ 9), cofeci (CIL $\left.\mathrm{I}^{2} 560\right)$. C'est le type scies (CIL I $\left.{ }^{2} 366\right)$ pour sciēns.

15 En raison de la disparition très ancienne de $/ \mathrm{m} /$ devant $/ \mathrm{w} /$ (cf. cōntiō < *ko[m]- $w^{\circ}$ ). Pour un phénomène parallèle en celtique (vieil irlandais cóir, co(a)ïr "approprié”), voir Martzloff (2012: pp. 85-86), avec bibliographie, en particulier Schrijver (1995: p. 326). Pareillement en sabellique: selon nous, les formes ombriennes eikvasatis (TIg III 24, 29) et eikvasese (TIg Va 4, 16) s'analysent non en * eik-vas ${ }^{\circ}$ (avec un $k$ suffixal), mais en $e i$ - $\left(k-v a s^{\circ}\right)$, avec syncope du /o/ dans le préfixe $k o$ - du second élément de composé. Or cette syncope n'a pu avoir lieu que si le préfixe n'avait pas (ou plus) de consonne nasale en proto-sabellique. Voir Martzloff (2012: p. 90). 
rement les mêmes. Comme l'amuïssement de la nasale dans / ns/ est achevé, l'accusatif deiuos ne peut pas servir à illustrer la non-notation d'une nasale simplement affaiblie.

- (3) Le nom Qetios (base de Tibur) a parfois été rattaché à Cottius, mais on peut aussi penser à l'ancêtre de Quinctius ${ }^{16}$ avec une lettre $Q$ transcrivant à elle seule une labiovélaire (cf. QOI et falisque iofete-qe dans $\operatorname{LinFa} 3)^{17}$ et surtout avec une réalisation [ $\left.{ }^{\mathrm{n}} \mathrm{t}\right] \mathrm{de} / \mathrm{nkt} /$ et $\eta$ non noté. L'emploi de $Q$ n'est donc pas un simple caprice d'orthographe. Dans cette forme ancienne de Queinctius, le groupe /nk/ (placé devant une occlusive) devait pouvoir être réalisé comme un unique son [n] (nasale vélaire, cf. Washington ['wpfintən]). Le contexte phonétique est certes différent de celui de OITES [oite ${ }^{\mathrm{n}} \mathrm{ss}$ ], mais la graphie qetios montrerait que, du point de vue orthographique, la non-notation d'une nasale (et même d'une nasale vélaire) était possible dès les $\mathrm{VI}^{\mathrm{e}}-\mathrm{V}^{\mathrm{e}}$ siècles. Nous proposons donc de mettre en rapport la non-notation de la nasale anté-consonantique dans qetios, dans cosmis et dans oites.

- (4) Le fragment d'une inscription latine archaïque de Satricum ]Xa mamarcom placiom [ (575-525) présente deux génitifs pluriels (ou deux accusatifs singuliers, peu importe ici), dont le second, placiom, n'a pas reçu d'explication satisfaisante. Adamik (2010: p. 25, note 34$)$ a pourtant suggéré une interprétation $P l a^{(n)}$ ciom, qui nous semble très prometteuse (cf. Plancius). Néanmoins, Adamik a cru devoir renoncer à cette intéressante analyse pour la raison que le /n/ anté-consonantique ne manquerait jamais dans les textes latins archaïques. Mais ce dogme rigide doit être abandonné. Selon nous, placiom pourrait présenter une omission d'une nasale vélaire [n], exactement comme qetios: $\left[\right.$ pla $\left.{ }^{\mathrm{n} k i o s}\right]$ serait parallèle à $\left[\mathrm{k}^{\mathrm{w}} \mathrm{e}^{\mathrm{n}} \mathrm{tios}\right]$ (ce [ $\left.\mathrm{k}^{\mathrm{w}} \mathrm{entios}\right]$ étant une réalisation phonétique de $/ \mathrm{k}^{\mathrm{w}}$ enktios/). Or placiom et qetios sont des noms propres, ce qui indique que le statut de nom propre n'était pas en soi un obstacle à l'omission graphique d'une nasale à époque ancienne. On posséderait donc quatre exemples d'omissions graphiques d'une nasale, deux d'une nasale vélaire (qetios, placiom) et deux d'une nasale dentale (cosmis, oites).

- (5) Le falisque a $f[i(:) f] i q o d(\operatorname{LinFa} 1)$ où $-O D$ transcrit $/ \mathrm{o}^{\mathrm{n}} \mathrm{d} /$, mais l'environnement phonétique n'est pas le même que dans oites (en fait, on ignore comment ce $D$ final paléo-falisque était réalisé) et on ne peut pas ici transposer mécaniquement les données falisques au latin de Rome, car le secteur falisque est trop éloigné de Rome pour que les habitudes orthographiques soient nécessairement comparables.

- (6) Plusieurs auteurs acceptent l'idée que le groupe écrit -PES (dans la séquence paléo-falisque fitaidupes de LinFa 1) représente $\left[\mathrm{pe}^{\mathrm{n}} \mathrm{ss}\right.$ ] $<$ *-pend-s (avec le même second élément que lībripēns). Si cette interprétation était correcte, elle fournirait un parallèle exact à oites. Néanmoins, nous ne voulons pas décider ici de la véracité de cette analyse. ${ }^{18}$

- (7) Falisque salueto (LinFa 3) provient-il de *saluēntōd? Voir Poccetti (2018: p. 350). Mais la nasale se trouverait devant l'occlusive / t/. Et le falisque n'est pas le latin de Rome.

16 V. Martzloff précise que le nom qetios avait fait l'objet d'un exposé à l'Université Lumière-Lyon II, il y a quelques années, par Alberto Calderini. Nous sommes les seuls responsables de l'interprétation donnée ci-dessus.

17 Le sigle LinFa (pour Lingua Falisca) renvoie à Giacomelli (1962). Voir Rix (1993: p. 86).

18 L'identification de $i d u$ - comme *endo- est très douteuse. Analyse alternative chez Martzloff (2014: p. 236; 2015: p. 97, note 181), avec bibliographie. 
- (8) Nous ne voulons pas ici décider si ofetios ( LinFa 3) remonte à *ouß-ent-io-s, comme le propose astucieusement Rix (1993: p. 86).

- (9) Un élément beaucoup plus intéressant que ces formes falisques nous est livré par la fameuse tessère d'hospitalité en langue étrusque trouvée à Rome dans le secteur de S. Omobono, portant une inscription datée d'entre 580 et 540: araz silqetenas spurianas (ET La 2.3), cf. Maggiani (2006: pp. 321, 325-327, 335, 341-342). Cet araz est généralement mis en relation avec $\operatorname{aran} \theta$, en supposant une affrication de $\theta(?)$ et une absence de nasale (nasale amuie, ou affaiblie et non notée?). Une autre inscription étrusque de Rome (ET La 2.4, $\mathrm{VI}^{\mathrm{e}}$ siècle) contient le génitif araziia. Même s'il s'agit d'étrusque, non de latin, la forme araz (cf. Arruns) nous autorise à admettre qu'une nasale anté-consonantique pouvait être omise dans la graphie à l'horizon chronologique de la première moitié du $\mathrm{VI}^{\mathrm{e}}$ siècle.

Finalement, avec les réserves qui s'imposent, ces remarques suggèrent que, par suite d'un phénomène aréal, une nasale anté-consonantique pouvait être déjà faiblement prononcée et non écrite dans le Latium et ses environs, en particulier à Rome, dès le $\mathrm{VI}^{\mathrm{e}}$ siècle. Cela serait encore plus aisé à admettre devant/ss/ double. Nous affirmons donc que l'analyse de oites [oite ${ }^{\mathrm{n}} \mathrm{ss}$ ] comme l'ancêtre du participe ūtêns proposée par Rudolph Thurneysen est inattaquable en soi, et qu'il serait tout simplement faux de considérer que l'absence de nasale dans la graphie est une objection. Du point de vue de la syntaxe, le participe oites est construit avec le cas ablatif (comme on l'attend): riuois. La disjonction oites... riuois possède un parallèle dans soc[iai]... karai (inscription paléo-falisque dite “de Cérès”, LinFa 1).

L'analyse de oites comme précurseur de $\bar{u} t \bar{e} n s$ (qui semble irréprochable) nous amène à une division oites / iai / paca (ou pacar) / riuois. Cette segmentation implique l'existence d'un mot écrit IAI. Selon nous, ce iai est un ancien locatif singulier féminin (figé en adverbe) appartenant au paradigme pronominal d'où procède iam. ${ }^{19}$ La même terminaison de locatif féminin singulier est attestée en sabellique dans la conjonction de subordination svai (svai, suae) de l'osque et suai du sud-picénien (dans suaipis, ST Sp TE 1). L'ombrien présente une monophtongaison: sve, sue. Le iai latin apparaît dans un système conditionnel (dont il introduit l'apodose), tandis que svaí introduit la protase d'un système conditionnel.

Le mot iai forme une équation exacte avec le premier élément de l'adverbe ombrien iepi, issu de *iya $i-k^{w} i d . .^{20}$ La proposition subordonnée ap vuku kukehes "quand tu approcheras du bosquet” (TIg III 20-21) est suivie de sa régissante introduite par l'adverbe

19 Le monosyllabe latin iam "déjà" (apparenté à iai) remonte probablement à une forme dissyllabique *iyām, qui a subi une réduction de l'initiale $* i y>* y$. La possibilité d'une réduction /iy/ > /y/ à l'initiale absolue du mot est confirmée par un examen de la préhistoire du verbe latin iuuāre "aider", dont le $i$-initial note une consonne: [yuw]. Le présent à redoublement $* h_{x} i-h_{x} u h_{y}$ - (avec deux laryngales $h_{x}$ et $h_{y}$ dont nous ne précisons pas ici la qualité) a évolué régulièrement en /iyuw/. Il faut donc admettre une réduction de /iyuw/ en /yuw/. Voir Weiss (2010: p. 145), qui reconstruit un prototype *iyāi de locatif en deux syllabes, et Martzloff (2018: p. 240).

20 Du point de vue de la phonétique historique, l'élément $i e$ - est certes susceptible de plusieurs explications, mais, comme le souligne Weiss, la reconstruction *iyāi est la plus satisfaisante. L'équation entre $i a i$ et $i e(p i)$ a été envisagée dans Martzloff (2015: p. 97; 2018: p. 238) et Machajdíková \& Martzloff (2016: p. 101). 
iepi, dont le sens serait "en chemin" ou "à un moment donné" ou "alors". La séquence nei ted endo cosmis uirco sied [...] oites iai paca riuois signifierait: "Si la jouvencelle n'était pas (nei... uirco sied) aimable (cosmis) envers toi (ted endo) [...], alors (iai) apaise-la (paca) en utilisant (oites) les écoulements (riuois)" [ou: "alors (iai) en [m'] employant (oites) apaise-la (paca) par les écoulements (riuois)"].

[Si on préfère extraire un subjonctif pacar d'un déponent thématique (cf. pac-īsc-or) au lieu de l'impératif paca, la première personne de pacar ferait référence au fournisseur du vase: “Celui qui m’offre jure par les dieux: 'si la jouvencelle n'était pas aimable envers toi [...], alors (iai) je [moi qui remets le vase] rétablirais la situation / je fixerais les conditions d'un contrat [de mariage?] (pacar) en utilisant (oites) les écoulements (riuois, libation solennelle?)'."]

\section{Le verbe opet et sa construction syntaxique}

Penchons-nous sur la portion de texte astednoisiopet. Harđarson (2011: p. 158) a proposé d'extraire un verbe opet qui serait apparenté à optāre "désirer". Harđarson et Rix \& Kümmel (2001: p. 299) reconstruisent une racine possédant une laryngale III $\left(h_{3}\right)$ initiale et expliquent opèt comme le reflet de *hзs op-éye-ti. Selon Weiss (2010: pp. 95-96), il est plus économique de poser $* h_{1}$ op-éye-ti, qui serait un présent itératif formé avec la racine *h epp-signifiant "prendre" (hittite èpzi, appanzi "saisir", latin apiscor). Cette incertitude concernant la laryngale est sans conséquence pour notre propos. Les deux reconstructions sont compatibles avec l'identification d'un indicatif présent opet "elle désire": $h_{1 / 3} o p$ éye-ti $>*$ *opeyet $i>*$ opeet $(i)>$ opēt (type moneō: *mon-éye-ti $>*$ moneet $(i)>$ monèt $>$ monet $)$. On remarquera que opet et sied sont positionnés à la fin des propositions dont ils sont les noyaux. On constate que les deux protases coordonnées par ast présentent des verbes conjugués à des modes différents: nei... sied (subjonctif), ast... opet (indicatif).

Un interprète de la séquence ast ted noisi opet doit se poser la question suivante: quels types de compléments est-on en droit d'attendre dans une proposition contenant un verbe opet signifiant "elle désire"? Cette question amène à envisager que opet est associé à deux types de compléments, un objet direct indiquant "ce que" le sujet réclame et un complément indiquant "à qui" ou "de qui” le sujet réclame une entité. En conséquence, nous suggérons que ted est un ablatif: ted ... opet "elle veut de toi" (cf. petere ab aliquō). Si on cesse d'analyser ted comme un accusatif, il faut chercher ailleurs l'objet direct. La séquence ne comporte pas de mot à l'accusatif. L'objet doit donc être identifié au seul segment disponible, qui est NOISI.

L'hypothèse que nous souhaiterions explorer consiste à analyser la lettre $I$ finale de noisi comme la marque du génitif singulier d'un thème nominal en /o/ (comme le gén. sg. domin̄ de dominus). ${ }^{21} \mathrm{Si}$ on acceptait d'admettre la présence d'un tel génitif à titre expérimental, il conviendrait de donner une justification sémantico-syntaxique précise

21 La terminaison -ī de génitif singulier remonte à *-iH (-i suivi d'une laryngale). Parmi les attestations anciennes, nous citerons: Aisclapi pococolom (CIL I $\left.{ }^{2} 440\right)$, Keri pocolom (CIL I $\left.{ }^{2} 445\right)$, Saeturni pocolom (CIL $\left.\mathrm{I}^{2} 449\right)$, Uolcani pocolom (CIL I $\left.{ }^{2} 453\right)$. 
à ce génitif. Or il existe des indices qui autorisent à penser qu'un verbe de désir transitif comme opet "elle réclame, elle exige, elle demande, elle désire, elle convoite" devait pouvoir se construire avec un complément d'objet au génitif en latin archaïque.

Assurément, Guardì (1974: p. 181) paraît exagérer quand il écrit: "il genitivo coi verbi di desiderio, normale in greco, non è raro in latino arcaico." En réalité, le génitif employé comme cas grammatical de l'objet d'un verbe de désir existe certes incontestablement en latin, mais il faut reconnaître qu'il est rarissime. ${ }^{22}$ Deux verbes méritent d'être cités, cupiō et studeō.

La construction de cupiō avec le génitif est moins souvent attestée qu'on ne le souhaiterait pour une argumentation telle que la nôtre. La réalité de cette construction est acceptée sans commentaire particulier par Bennett (1914: p. 98). On écartera Plaut. Mil. 963, où le génitif tuī (cupiunt tui) est une conjecture. On trouve quamquam domi cupio, opperiar (Plaut. Trin. 842) "malgré mon envie d'être à la maison, j'attendrai": tel est le texte de A et de Donat (ad Ter. Eun. 4, 7, 45 [815]), alors que les autres manuscrits ont domum. Mais ce domì est-il bien un génitif? Un génitif domī est attesté ailleurs (cf. domi focique dans le passage cité de Térence): les sceptiques auraient donc tort de vouloir retrouver ici un locatif employé dans une tournure elliptique. Beaucoup plus tard, on trouve une attestation de cupiō avec le génitif chez Apulée (Flor. 14), mais dans une construction au passif: Adeoque eius cupiebatur, ut uirgo nobilis [...] ultronea eum sibi optauerit "Et telles étaient les passions qu'il [Cratès] inspirait, qu'une fille de haute naissance [...] lui fit les premières avances". Schmalz (1905: p. 366) traduit "und so sehr war man in ihn verliebt". Faut-il y voir un emploi archaïsant imité du théâtre républicain?23

Outre la construction de cupiō avec le génitif, il reste le cas intéressant de studeō, qui est construit avec le génitif chez Caecilius Statius (Com. 199-201 Ribbeck, cité par Cicéron dans le De natura deorum, 3, 29, 72): In amore suaue est summo summaque inopia / parentem habere auarum inlepidum, in liberos / difficilem, qui te nec amet nec studeat tui "quand on est très amoureux et sans la moindre ressource, il est doux d'avoir un père avare, revêche, dur envers ses enfants, qui ne t'aime pas et ne s'intéresse pas à toi."

D'autres langues illustrent la construction au génitif des verbes de désir. Pour le grec

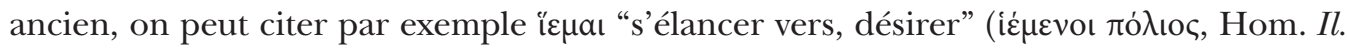
11, 168) et $\dot{\varepsilon} \pi \theta u \mu \dot{\varepsilon} \omega$ "désirer" (Her. 2, 66; Aesch. Ag. 214-217). Pour le lituanien, Seržant (2014: pp. 289-290) met en relation la construction au génitif des verbes de volonté ou de désir (comme ieškoti "chercher, to look for", noreiti "vouloir") avec le fait que ces verbes appartiennent à la catégorie des intensional verbs.

En outre, la construction au génitif est bien attestée pour des verbes latins exprimant un manque (un domaine sémantique proche de celui du désir): si pudoris egeas (Plaut. Amph. 819), neque consili neque audaciae umquam eguere (Sall. Catil. 51, 37), huius indigeas

22 Schmalz (1905: p. 366) considère que l'emploi du génitif objet résulte d'une simple imitation d'une structure nominale cupiens sum alicuius. Il est difficile d'accepter une conception aussi mécaniste des contaminations syntaxiques. Selon Pinkster (2015: p. 116), le génitif objet de cupere exprime les "cause or source of the emotion". 
patris (Ter. Andr. 890), nostri non indigerent (Rhet. Her. 3, 29), tui carendum (Ter. Haut. 400), 'carendum tui est' pro 'te' (Gell. 19, 7, 7, citant Laevius Carm. frg. 12 Blänsdorf).

D’un strict point de vue méthodologique, les données latines (studeat tui, domi cupio), grecques et lituaniennes accréditent l'idée qu'il est légitime de prendre en considération la possibilité que le verbe de désir opet (apparenté à optāre) puisse être construit avec un génitif objet. Afin d'identifier le lexème écrit noisi, il faut s'aider du contexte pour tâcher de mieux cerner sa signification. Le destinataire du vase (duenoi) reçoit la consigne d'“apaiser" (paca) la jouvencelle en utilisant $\left(\right.$ oite $\left.^{(n)} s\right)$ les "écoulements" d'un élément liquide (riuois). Cela pourrait impliquer que la nénette éprouve une certaine convoitise pour ce liquide. Nous voulons précisément suggérer que noisi est le génitif singulier d'un substantif désignant un liquide. Le génitif partitif s'expliquerait d'autant mieux (cf. impleto aquae purae, Cat. Agr. 88, 1). Or il existe une racine verbale qui conviendrait bien pour expliquer à la fois la morphologie et la sémantique de noisi. Selon nous, noisi représente /noissī/, qui serait le génitif singulier d'un substantif *noissos reflétant une

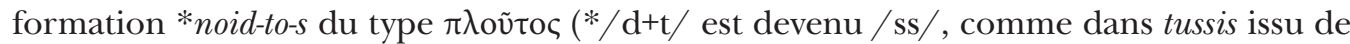
*tud-ti-s), contenant la même racine que le verbe védique nédati "il s'écoule, il déborde".

\section{Présentation de la racine "neid- (védique nédati, hydronymes "nidā, "neidā)}

La distribution des attestations actuellement connues de la racine *neid- est très singulière: cette racine est attestée dans les langues indo-iraniennes et dans l'hydronymie de l'Europe. Le sanskrit offre un verbe nédati interprété comme "fließen, strömen, überströmen, überfließen”. ${ }^{24}$ Une double attestation nous est donnée par exemple dans la Bṛhad-Āranyaka Upanișad, où sont distingués trois types d'offrandes: ya hutā ujjualanti, yā hutā atinedanti, yā hutā adhiśerate "celles qui flambent, celles qui débordent, celles qui s'étalent". Le texte enseigne que "par celles qui débordent, c'est le monde des mânes qu'on obtient" (yā hutā atinedanti, pitr-lokam eva tābhir jayati). ${ }^{25}$

La racine *neid- apparaît dans les langues iraniennes. Le khotanais a gganīh- "humecter, mouiller." ${ }^{26}$ Cheung (2007: pp. 277-278) cite également le verbe baloutchi nidit/nid"to cause to overflow, make brimful" et conclut: "Although the evidence for an Ir. root *naid is sparse, it can be established quite firmly, being attested in an East and a West Ir. language".

On trouve de nombreuses traces de la racine *neid- "couler" parmi les hydronymes (et les toponymes) européens. Une liste peut en être établie en consultant la monographie de J. Udolph. ${ }^{27}$ Il convient naturellement de rappeler les incertitudes qui pèsent

24 Gotō (1987, p. 202), Mayrhofer (1992-2001: II, p. 55), Rix \& Kümmel (2001: p. 449), Werba (1997: p. 430).

25 BrhUp 3, 1, 8. Texte et traduction d'après Senart (1934: p. 43).

26 Bailey (1960: pp. 21-24). Cf. Bailey (1979: p. 79).

27 Udolph (1990: pp. 181-185), avec carte (p. 186).Voir aussi les travaux de Rudnicki (1930: p. 699), Krahe (1959: p. 226; 1964: p. 48), Villar (1996: pp. 165-170), Toporov (1988: p. 162), Vasil'ev (2009: pp. 268269). Il s'agit de l'une des racines les plus étudiées en matière d'hydronymie: cette racine est même citée 
sur l'étymologie des noms de lieu en général (surtout quand ceux-ci ont été enregistrés à une date tardive). Néanmoins, même si l'analyse de tel ou tel nom propre devait être contestée, il paraît difficile de les rejeter tous et de nier l'emploi de la racine *neid- dans l'hydronymie européenne. Plusieurs savants estiment que ces noms ont été attribués dans le cadre d'un substrat alteuropäisch: explicitement Udolph (1990: p. 185); voir récemment Strandberg (2016: p. 109). La liste suivante ne vise pas à l'exhaustivité, mais se veut représentative.

Dans les Îles Britanniques, on trouve: (1) Neth, Neet (ou Strat, en Cornouailles, près de Bude); - (2) Neath (Afon Nedd) et le toponyme Nido sur l'Itinéraire d'Antonin (Cuntz 1990: p. 74); - (3) Nidd, affluent de la rivière Ouse (Yorkshire). Ces noms autorisent la reconstruction d'un degré zéro *Nid- dans la proto-forme.

En Europe continentale, on trouve une série d'attestations dont un sous-ensemble est centré dans la région du Bénélux, mais déborde au sud (Lorraine) et au Nord (Scandinavie méridionale): (4) Nied, nom attribué à deux cours d'eau, la Nied française (source à Marthille) et la Nied allemande (source à Seingbouse et Guenviller), formant la Nied Réunie, affluent de la Sarre; - (5) Nèthe (néerlandais Nete), rivière belge, affluent du Rupel: il existe une Grande Nèthe et une Petite Nèthe, ainsi que des affluents et sous-affluents appelés Neethje, mais une forme ancienne, écrite Huita, corrigée en Hnita, présente un $h$ - initial (Vincent 1925: p. 73); - (6) Nidda, affluent du Main, et toponyme Nida (Heddernheim, près de Frankfurt am Main), cf. CIL XIII 7263, 11944: voir Rasch (2005: pp. 75-76) et Kerneis (1999: pp. 368, 375, 390); - (7) Nethe, affluent de la Visurge (Weser), dans le Kreis Höxter; - (8) Nitja (Norvège).

Un nombre élevé d'attestations est disséminé en Pologne, en Lituanie, en Lettonie, en Biélorussie et en Russie occidentale: (9) Nida, rivière du sud de la Pologne, affluent gauche de la Vistule (Wisła, Weichsel), dans laquelle elle se jette près de la ville de Nowy Korczyn, au nord d'Opatowiec, lieu de confluence avec le Dunajec; - (10) Nidzi$c a$, affluent de la rive gauche de la Vistule, à quelques kilomètres au sud d'Opatowiec (Rozwadowski 1948: p. 295); - (11) Nida (all. Neide), nom pris par la rivière polonaise Wkra (affluent du Narew et sous-affluent de la Vistule) sur une partie de son cours, depuis la source non loin de Nidzica (Neidenburg) dans la voïvodie de Varmie-Mazurie (Województwo Warmińsko-Mazurskie), jusqu'à ce qu'elle soit rejointe par la Szkotówka (Skottau) près de Działdowo (Soldau), après quoi elle est nommée Dziatdówka (Soldau également), avant de recevoir le nom de $W k r a{ }^{28}$ Rozwadowski (1948: p. 295) voulait rapprocher la famille de l'adjectif polonais niski "bas", en comparant le $-d$ final de pod et zad, mais cela est difficile; - (12) Nida, ruisseau près de Gąbin; - (13) ville de Ruciane-Nida (Pologne, voïvodie de Varmie-Mazurie) avec un lac nommé Jezioro Nidzkie et un ruisseau appelé Nidka; - (14) localité polonaise de Nidowo (Niedau); - (15) hydronymes lituaniens Niedà, Niedùs et Niẽdis, ainsi que Niẽdulis, Niẽdulè $;{ }^{29}-(16)$ Schmid (1989: pp. 29-30) aborde les problèmes que suscite le rattachement du toponyme Nida

comme exemple dans l'article introductif de Strandberg (2016: p. 109).

28 Nous avons consulté, complété et remanié les informations données par Preuß (1835: pp. 31, 54).

29 Otrębski (1949: p. 148), Vanagas (1981: p. 230), Bichlmeier (2019: pp. 204-205, 223). 
(Nidden, avec brève) de Courlande à la famille de nédati; - (17) Niedin-ęzęrs, Lettonie: Vanagas (1981: p. 230); - (18) Nidal'ka, affluent droit de la Bérézina (Biélorussie); - (19) Nidel'ka ou Nedel'ka (bassin de la rivière Sož'). D'autres formes peuvent être relevées, mais il est parfois difficile d'évaluer leur pertinence. L'apparentement entre les formes de Grande-Bretagne et de Pologne avait déjà été proposé par Ferguson (1862: p. 54), mais avec un rattachement à une racine différente. Le rapprochement avec le verbe nédati se trouve chez Loewenthal (1930: p. 81) et Pokorny (1940: p. 98).

La Péninsule Ibérique a livré plusieurs formes pouvant contenir la racine de nédati. (20) Le théonyme Reue est associé à un adjectif Langanidaeigui (Portugal, Castelo Branco), qui est fléchi au datif singulier et dont on trouve une variante écrite Langanitaeco. ${ }^{30}$ Le $T$ du segment NITAECO a été interprété avec des arguments plausibles comme une hypercorrection dans un processus de latinisation. En effet, sur le modèle du $C$ transposant, dans une graphie davantage latinisée, la sonore notée $G$ (elle-même issue de la lénition d'un / $\mathrm{k}$ / plus ancien) apparaissant dans la forme proprement indigène, la dentale sonore originelle a pu être transposée abusivement en $T$, comme le suggère Villar (1996: pp. 167-168). Le premier élément écrit $L A N G A$ a suscité plusieurs hypothèses, parmi lesquelles on peut envisager, suivant Prósper (2002: pp. 134-135), une comparaison avec différents lexèmes balto-slaves, la famille de tchèque louka, slovaque lúka "prairie" (avec occlusive sourde) ${ }^{31}$ ou la famille de russe ljága "mare, marais, marécage" (avec occlusive sonore).$^{32}$ Ce mot lusitanien complexe s'interprète peut-être comme un composé copulatif unissant deux noms propres de cours d'eau: "relatif à / appelé la Langa (et) la Nida" (Prósper 2002: p. 136). - (21) Une forme NIDA (Bembibre, León) est également attestée (Prósper 2002: p. 137). - (22) Enfin, Prósper (2002: p. 234) ajoute l'épithète Nedoledio, en supposant un degré plein: *neid-ol/ul-et/êt-iyo-s (?).

\section{Analyse phonologique et morphologique de noisi}

Nous interprétons NOISI comme /noissī/, génitif singulier d'un lexème *noissos issu de *noid-to-s, appartenant au même type morphologique que le mot grec koĩtoৎ (schème $* C_{1} \delta C_{2}$-to-s). Suivant García Ramón (2016), les formations du type $* C_{1} \delta C_{2}$-to-s constituent en grec un groupe peu productif qui provient lui-même du renouvellement formel d'autres

30 Formes recensées chez Blázquez (1983: p. 485) et Untermann (1985: pp. 351, 359). Schmoll (1959: p. 34) posait, à tort, un gentilice * Langanitaikos.

31 Cette famille couvre un spectre sémantique assez vaste: lituanien lankà "der Überschwemmung ausgesetzte Wiese, Aue, Marsch, Tal" (Fraenkel 1962-1965: I, p. 339), russe luká "Biegung, Krümmung, Wiesen- oder Waldland einer Flußkrümme, Sattelbogen" et vieux slave lqka "List, Betrug". Voir Vasmer (1953-1958: II, p. 68) et Králik (2015: p. 335). Cette divergence sémantique est due au fait que ces mots se rattachent à la famille du verbe leñkti, lenkiù "courber" (Fraenkel 1962-1965: I, pp. 356-357), avec une évolution sémantique de "hollow, depression" à "low-lying meadow, water-meadow", selon Derksen (2015: pp. 272-273).

32 Vasmer (1953-1958: II, p. 80). Cf. lituanien lénge "tiefer gelegenes Stück Land, Vertiefung bildende kleine Wiese, Wieschen zwischen zwei Anbergen" (Fraenkel 1962-1965: I, p. 355) et russe lúg "Wiese, Aue" (Vasmer 1953-1958: II, pp. 64-65). Voir Derksen (2008: pp. 288-289; 2015: pp. 279-280). Trautmann (1970: p. 158) mentionne un nom letton de ruisseau Langa et un nom prussien de marais Langodis. 


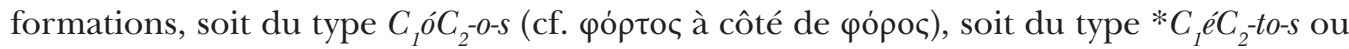

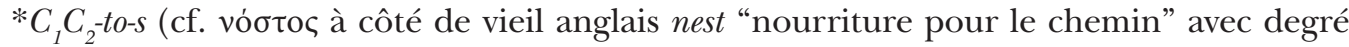
/e/ et védique ásta- "résidence, foyer" avec degré zéro).

Ce type morphologique est certes rare en dehors du grec, mais il est indéniablement

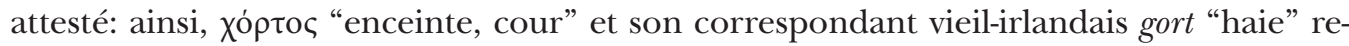
montent à * $\hat{g}^{h}$ ór-to-s. Latin hortus et osque húrz, húrtúi, húrtúm, húrtín peuvent refléter un degré zéro ou un degré /o/, mais le degré /o/ est préférable, car il autorise une

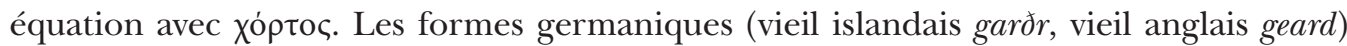
sont ambiguës, car la consonne qui suit le /r/ peut remonter à */t/ ou à */ $\mathrm{d}^{\mathrm{h}} /(\mathrm{cf}$.

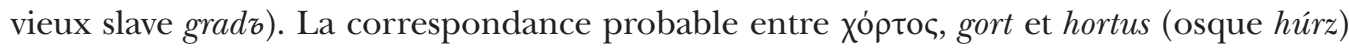
montre que le type morphologique $* C_{1} o C_{2}$-to-s peut avoir eu une certaine ancienneté, au moins dans quelques lexèmes.

En dehors de hortus et peut-être de *noissos < *noid-to-s (dans NOISI), les langues italiques pourraient posséder une formation du type $* C_{1} \delta C_{2}$-to-s dans la forme "sicule" $\mu$ oĩ par Varron et Hésychius, dans le dénominatif latin mūtāre et le dérivé mūtuus. En réalité, hélas, l'analyse de $\mu$ oĩ gensatz, gegenseitig") ou *(h, $\left.h_{2}\right)$ mó-to-s (racine de commūnis, gotique gamains, vieux slave měna). Ainsi, Vine (1999: p. 297) reconstruit *mói-to-s "something given in exchange, tit for tat".

Un parallèle remarquable à *noid-to-s est fourni par le lexème grec $\pi \lambda$ oṽ qui est une formation ancienne à l'échelle de la langue grecque (dérivé mycénien po-

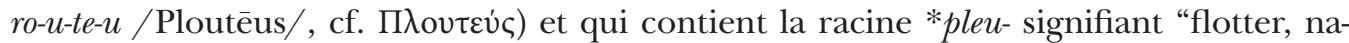

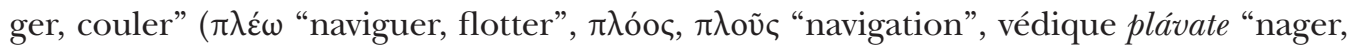
flotter", latin pluit "il pleut"). La signification de $\pi \lambda$ oṽ $\tau$ oৎ repose sur la métaphore de l'abondance, qui est attestée dans plusieurs langues: latin adfluēns "abondant" (fluō "couler, s'écouler"), abundāre "déborder, être abondamment pourvu de" (unda "eau"), grec

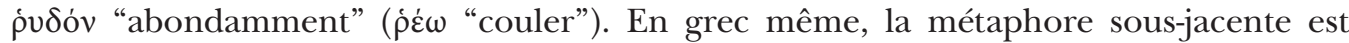

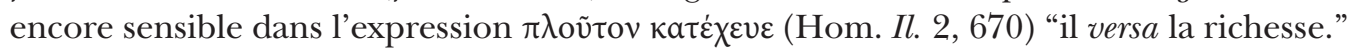

En outre, Willi (2004: p. 332) interprète ä $\varphi \varepsilon v o \varsigma$ "richesse, abondance” comme un dérivé d'une racine $* h_{2} e b^{h}$-apparaissant, selon lui, dans palaïte hăpna- et hittite hapa- "rivière", vieil irlandais $a b$ (génitif $a b a e$, d'un thème en $/ \mathrm{n} /)$ et peut-être latin amnis $\left(<* h_{2} e^{-}\right.$ $b^{h}-n-i-s$ ?). [Bien sûr, la sonore de certaines formes a parfois été expliquée comme résultant d'une sonorisation secondaire due au suffixe de Hoffmann $*_{-} h_{3} e n$ - (si, du moins, on admet que celui-ci contenait une laryngale III): voir la bibliographie et la discussion chez Pinault (2001: p. 97). Ainsi, $e$ DiAna ${ }^{33}$ ne favorise pas la reconstruction de $* h_{2} e b^{h}$-avec une sonore aspirée. Néanmoins, la sonore se rencontre également dans des formes anatoliennes sans nasale, comme le souligne Willi (2004: p. 331, note 9), selon qui une racine avec $* b^{h}$ final serait confirmée par le mot grec. Et Cisauna $\left(\right.$ CIL I $\left.{ }^{2} 7\right)$ reflète-t-il sabellique $*_{c i s}+a b^{h} n \bar{a}$ ? Ces analyses semblent appuyées par l'expression jodòv à $\varphi v \varepsilon$ ioio (Hom.

33 https://www.ediana.gwi.uni-muenchen.de/dictionary.php?lemma=352 (consulté le 20 mars 2020). La forme ha-ap-pa (KUB XXXI 74 II 9) paraît impliquer $* h_{2} e p$-: voir Eichner (1974: p. 215); la question est de savoir s'il faut opérer uniquement avec cette racine, ou s'il faut admettre les deux racines $* h_{2} e p^{-}$et $* h_{2} e b^{h}$ - en anatolien: discussion chez Puhvel (1991: p. 115), avec bibliographie. 


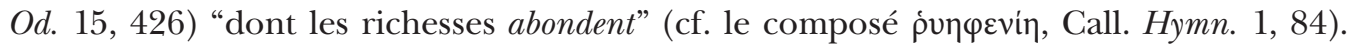

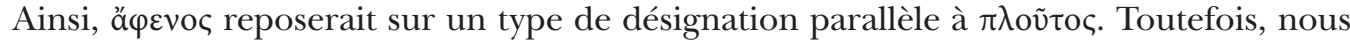
ne voulons pas insister ici sur l'analyse diachronique de ä $\varphi \varepsilon v o \varsigma$, qui est sans grande incidence sur notre argumentation d'ensemble.] On peut donc établir les correspondances suivantes:

\begin{tabular}{|c|c|c|}
\hline $\begin{array}{l}\text { Racine } \\
\text { Sens de la racine } \\
\text { Formation de type } \chi \text { ó } \rho \tau o \varsigma \\
\text { Forme attestée }\end{array}$ & 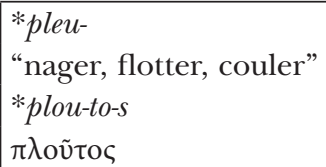 & $\begin{array}{l}\text { *neid- } \\
\text { "couler à flots" } \\
\text { *noid-to-s > *noissos } \\
\text { génitif noisi / noissī/ }\end{array}$ \\
\hline
\end{tabular}

L'évolution phonétique $/ \mathrm{d}+\mathrm{t} />/ \mathrm{ss} /$ est comparable à celle qu'illustrent fissus (findō), scissus (scindō), et, avec une dégémination après voyelle longue, cāsus "chute, hasard, acci-

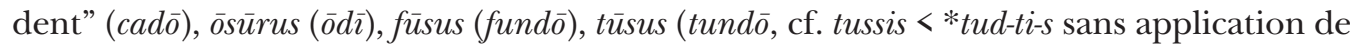
la loi de Lachmann), èsus (ed̄̄), uīsus (uidē̄). Le supin sessum est d'analyse controversée (remaniement en *sezdsum, non *sedsum?). On consultera Jasanoff (2004). Citons encore caesim (caedō; caestus "ceste" appelle une autre analyse).

En conclusion, notre interprétation de NOISI comme le génitif singulier d'un mot *noissos, venant de *noid-to-s (racine de védique nédati "couler à flots") et fonctionnant comme l'objet de opet "elle désire", paraît possible sur les plans phonétique (tussis < *tud$t i-s$ ), morphologique (hortus, v. irl. gort), sémantique ( $\pi \lambda$ oṽ $\tau$ ç) et syntaxique (studeat tuī, domi cupiō). Cette analyse permet de traduire la macro-séquence textuelle nei ted endo cosmis (*kon-sm ${ }^{\circ}$, non **ko-sm ${ }^{\circ}$ ) uirco sied, ast ted (abl.) noisi (gén.) opet, oites (ūtēns, cf. qetios [ $\mathrm{k}^{\mathrm{w}}$ entios], peut-être placiom, araz) iai (cf. ombr. iepi <*iya $\left.i-k^{w} i d\right)$ paca riuois:

"Si la jouvencelle n'était pas (nei... uirco sied) aimable (cosmis) envers toi (ted endo), mais (ast) désire / exige / réclame (opet) de toi (ted, abl.) du produit-liquide (noisi), alors (iai) satisfais-la / apaise-la (paca, cf. all. befriedigen) en employant (oites) des écoulements / des émissions, des décharges liquides (riuois)."

Le mot riuois qui termine la séquence fait système avec noisi. L'humour du passage réside dans l'identification (laissée ouverte) des référents de ces mots: aromates? parfums? cosmétiques? ou interprétation grivoise pour le terme riuois ${ }^{34}$ qui désignerait des épanchements destinés à combler la môme insatisfaite, qui est encore pucelle (uirco)? L'inscription du duenos, qui n'est nullement votive, se distinguait donc par un esprit badin et enjoué, chargé de sous-entendus érotiques, dans le ton de la fescennīna iocātiō (Catulle $61,127)$.

34 La métaphore implicite serait la même que dans le mot védique rétas- qui sert à désigner la liqueur séminale, si ce mot est bien apparenté à rïuus, riuois. Discussion de la famille védique chez Vijūnas (2008: p. 14). 


\section{Bibliographie}

Adamik, B. (2010). Zu den archaischen lateinischen Inschriftfragmenten auf Bruchstücken eines Tonfasses von Satricum. In P. Anreiter, \& M. Kienpointner (Eds.), Latin Linguistics Today (pp. 17-30). Innsbruck: Institut für Sprachen und Literaturen der Universität Innsbruck.

Bailey, H. W. (1960). 4. ganīh-. Bulletin of the School of Oriental and African Studies, 23(1), 21-24.

Bailey, H. W. (1979). Dictionary of Khotan Saka. Cambridge - London - New York - Melbourne: Cambridge University Press.

Bakalakis, G. (1991). Les kernoi éleusiniens. Kernos, 4, 105-117.

Benndorf, O. (1900). Note 3 (sans titre). Jahreshefte des Österreichischen Archäologischen Institutes in Wien, 3, 9-10.

Bennett, C. E. (1914). Syntax of Early Latin, II: The Cases. Boston: Allyn and Bacon / Leipzig: Stauffer.

Bichlmeier, H. (2019). Morphologische und semantische Analyse der Gewässernamen des westlichen Südaukštaiten (Teil 2). Acta Linguistica Lithuanica, 80, 195-263.

Blázquez, J. M. (1983). Primitivas religiones ibéricas, II: Religiones prerromanas. Madrid: Ediciones Cristiandad.

Bréal, M. (1882). L'inscription de Duenos. Mélanges d'archéologie et d'histoire, 2, 147-167.

Brugmann, K. (1909). Altitalische Miszellen. Indogermanische Forschungen, 24, 72-86.

Cheung, J. (2007). Etymological Dictionary of the Iranian Verb. Leiden - Boston: Brill.

Coarelli, F. 1988. Il Foro Boario. Dalle origini alla fine della Repubblica. Roma: Quasar.

Colonna, G. (1979). Duenos. Studi Etruschi, 47, 163-172.

Cristofani, M. (1990). Vaso di Duenos. In M. Cristofani (Ed.), La Grande Roma dei Tarquini, catalogo della mostra (pp. 20-21). Roma: "L'Erma" di Bretschneider.

Cuntz, O. (1990). Itineraria Antonini Augusti et Burdigalense. Stuttgart: Teubner.

Derksen, R. (2008). Etymological Dictionary of the Slavic Inherited Lexicon. Leiden - Boston: Brill.

Derksen, R. (2015). Etymological Dictionary of the Baltic Inherited Lexicon. Leiden - Boston: Brill.

Dressel, E. (1880). Di una antichissima iscrizione latina graffita sopra vaso votivo rinvenuto in Roma. Annali dell'Instituto di Corrispondenza Archeologica, 52, 158-195.

Ducati, P. (1938-1939). Sui primi tre documenti di lingua latina. Rendiconto delle Sessioni della R. Accademia delle Scienze dell'Istituto di Bologna, Classe di scienze morali, Serie IV, 2, 11-18.

Dumézil, G. (1969). Idées romaines. Paris: Gallimard.

Dumézil, G. (1979). Mariages indo-européens. Paris: Payot.

Dunkel, G. E. (2014). Lexikon der indogermanischen Partikeln und Pronominalstämme, Band 2, Lexikon. Heidelberg: Winter.

eDiAna: Digital Philological-Etymological Dictionary of the Minor Ancient Anatolian Corpus Languages [on line: https://www.ediana.gwi.uni-muenchen.de/].

Eichner, H. (1974). Untersuchungen zur hethitischen Deklination. Inaugural-Dissertation (Teildruck). Erlangen - Nürnberg.

Eichner, H. (1988-1990). Reklameiamben aus Roms Königszeit. Die Sprache, 34, 207-238.

Ferguson, R. (1862). The River-Names of Europe. London - Edinburgh: Williams \& Norgate.

Fraenkel, E. (1962-1965). Litauisches etymologisches Wörterbuch. Heidelberg: Winter / Göttingen: Vandenhoeck \& Ruprecht. 
Fraser, A. D. (1932). The inscribed kernos of Duenos. The American Journal of Philology, 53(3), 213-232.

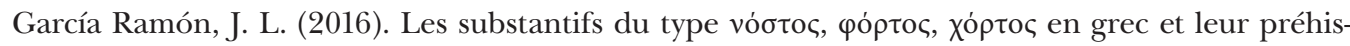
toire. In A. Blanc, \& D. Petit (Eds.), Nouveaux acquis sur la formation des noms en grec ancien (pp. 203-224). Leuven - Paris: Peeters.

Garnier, R. (2017). Scripta Selecta. Les Cent Chemins.

Giacomelli, G. (1962). La lingua falisca. Firenze: Olschki.

Godel, R. (1962). Note sur l'inscription du "vase de Duenos" (CIL I² 4). Cahiers Ferdinand de Saussure, 19, 101-106.

Goldmann, E. (1926). Die Duenos-Inschrift. Heidelberg: Winter.

Gordon, A. E. (1975). Notes on the Duenos-Vase Inscription in Berlin. California Studies in Classical Antiquity, 8, 53-72.

Gotō, T. (1987). Die "I. Präsensklasse" im Vedischen. Wien: Verlag der Österreichischen Akademie der Wissenschaften.

Götze, A. (1923). Relative Chronologie von Lauterscheinungen im Italischen. Indogermanische Forschungen, 41, 78-149.

Grienberger v., T. (1900). Zur Duenosinschrift. Indogermanische Forschungen, 11, 342-343.

Guardì, T. (1974). Cecilio Stazio. I frammenti. Palermo: Palumbo.

Harđarson, J. A. (2011). The $2^{\text {nd }}$ Line of the Duenos Inscription. Alessandria, 5, 153-163.

Hempl, G. (1902). The Duenos Inscription. Transactions and Proceedings of the American Philological Association, 33, 150-169.

Hencken, H. (1968). Tarquinia, Villanovans and Early Etruscans. Cambridge (Mass.): Peabody Museum.

Jasanoff, J. H. (2004). Plus ça change...: Lachmann's Law in Latin. In J. H. W. Penney (Ed.), IndoEuropean Perspectives. Studies in Honour of Anna Morpurgo Davies (pp. 405-416). Oxford: Oxford University Press.

Kerneis, S. (1999). La Bretagne rhénane. Note sur les établissements bretons dans les Champs Décumates. Latomus, 58(2), 357-390.

Krahe, H. (1959). Eigennamen und germanische Lautverschiebung. In Mélanges de linguistique et de philologie, Fernand Mossé in memoriam (pp. 225-230). Paris: Didier.

Krahe, H. (1964). Unsere ältesten Flussnamen. Wiesbaden: Harrassowitz.

Králik, L. (2015). Stručný etymologický slovnik slovenčiny. Bratislava: Veda.

Lipp, R. (2009). Die indogermanischen und einzelsprachlichen Palatale im Indoiranischen. Band II. Heidelberg: Winter.

Loewenthal, J. (1930). Etymologische Miszellen. Zeitschrift für Ortsnamenforschung, 6, 76-81.

Machajdíková, B., \& Martzloff, V. (2016). Le pronom indéfini osque pitpit "quicquid" de Paul Diacre à Jacob Balde. Graeco-Latina Brunensia, 21(1), 73-118.

Maggiani, A. (2006). Dinamiche del commercio arcaico: le tesserae hospitales. Annali della Fondazione per il Museo "Claudio Faina", 13, 317-349.

Martzloff, V. (2012). Sur la polymorphie du préfixe latin con- / co-. In A. Christol, \& O. Spevak (Eds.), Les évolutions du latin (pp. 67-103). Paris: L'Harmattan.

Martzloff, V. (Rev.). (2014). Compte rendu de: A. Mercado, Italic Verse (Innsbruck, 2012). Wék ${ }^{w}$ os, 1, 234-242. 
Martzloff, V. (2015). La plus ancienne composition poétique à Rome. L'inscription latine archaïque du duenos (CIL I² 4). Revue des Études Latines, 93, 69-106.

Martzloff, V. (2018). Métrique italique archaïque. Poésie sud-picénienne et inscription latine de Duenos. In D. Gunkel, \& O. Hackstein (Eds.), Language and Meter (pp. 222-252). Leiden - Boston: Brill.

Martzloff, V. (2019). Sur l'origine et la morphologie de quelques grammèmes de négation latins: ni, non, noenum, nisi. In M. Taillade, J. Gallego, F. Fatello, \& G. Gibert (Eds.), Nemo par eloquentia. Mélanges de linguistique ancienne en hommage à Colette Bodelot (pp. 109-124). ClermontFerrand: Presses universitaires Blaise Pascal.

Marzoli, D. (1989). Bronzefeldflaschen in Italien. München: C.H. Beck.

Mattiacci, S. (1994). Note sulla fortuna di Accio in Apuleio. Prometheus, Rivista quadrimestrale di studi classici, 20(1), 53-68.

Mayrhofer, M. (1992-2001). Etymologisches Wörterbuch des Altindoarischen. Heidelberg: Winter.

Meringer, R. (1907). Wörter und Sachen. V. Indogermanische Forschungen, 21, 277-314.

Montelius, O. (1904). La civilisation primitive en Italie depuis l'introduction des métaux. Deuxième partie, Italie Centrale, Planches, 2. Stockholm: Imprimerie Royale / Berlin: Asher \& Co.

Munro, R. (1890). The Lake-Dwellings of Europe, being the Rhind lectures in archaeology for 1888. London - Paris - Melbourne: Cassell.

Otrębski, J. (1949). Les mots d'origine commune dans les langues slaves et baltiques. Lingua Posnaniensis, 1, 121-151.

Pinault, G.-J. (2001). Le type latin uorāgō: un reflet d'un suffixe indo-européen. Glotta, 77, 85-109. Pinault, G.-J. (2013). Le vocabulaire et l'image du sourire dans les langues indo-européennes. In P.-S. Filliozat, \& M. Zink (Eds.), Sourires d'Orient et d'Occident (pp. 17-45). Paris: AIBL.

Pinkster, H. (2015). The Oxford Latin Syntax (Vol I). Oxford: Oxford University Press.

Pinza, G. (1905). Monumenti primitivi di Roma e del Lazio antico. Monumenti Antichi, 15, 5-798.

Poccetti, P. (2009). Paradigmi formulari votivi nelle tradizioni epicoriche dell'Italia antica. In J. Bodel, \& M. Kajava (Eds.), Dediche sacre nel mondo greco-romano. Diffusione, funzioni, tipologie (pp. 43-93). Roma: Institutum Romanum Finlandiae.

Poccetti, P. (2018). The -tōd Imperative in Italic Languages: Comparative and Typological Insights. In D. Gunkel, S. W. Jamison, A. O. Mercado, \& K. Yoshida (Eds), Vina Diem Celebrent. Studies in Linguistics and Philology in Honor of Brent Vine (pp. 346-362). Ann Arbor - New York: Beech Stave Press.

Poccetti, P. (2019). Oscan *deivāom 'to swear'. In N. Bolatti Guzzo, \& P. Taracha (Eds.), “And I Knew Twelve Languages". A Tribute to Massimo Poetto on the Occasion of His 70th Birthday (pp. 510-521). Warsaw: Agade Bis.

Pokorny, J. (1940). Zur Urgeschichte der Kelten und Illyrier. Zeitschrift für celtische Philologie, 21, 55-166.

Preuß, A. E. (1835). Preußische Landes- und Volkskunde oder Beschreibung von Preußen. Königsberg: Bornträger.

Prosdocimi, A. L. (1979). Studi sul latino arcaico. Studi Etruschi, 47, 173-221.

Prosdocimi, A. L. (2010). La Roma 'Tarquinia' nella lingua: forme e contenuti tra il prima e il dopo. Annali della Fondazione per il Museo «Claudio Faina», 17, 367-489.

Prósper, B. M. (2002). Lenguas y religiones prerromanas del occidente de la Península Ibérica. Salamanca: Ediciones Universidad de Salamanca. 
Puhvel, J. (1991). Hittite Etymological Dictionary, 3: Words beginning with H. Berlin - New York: Mouton de Gruyter.

Rasch, G. (2005). Antike geographische Namen nördlich der Alpen. Ed. S. Zimmer, \& H. Heiland. Berlin - New York: de Gruyter.

Rix, H. (1985). Das letzte Wort der Duenos-Inschrift. Münchener Studien zur Sprachwissenschaft, 46, 193-220.

Rix, H. (Rev.). (1993). Compte rendu de: La civiltà dei Falisci (Firenze, 1990). Kratylos, 38, 83-87.

Rix, H. (1999). Schwach charakterisierte lateinische Präsensstämme zu Seț-Wurzeln mit Vollstufe

I. In H. Eichner, \& H. C. Luschützky (Eds.), Compositiones Indogermanicae in memoriam Jochem Schindler (pp. 515-535). Praha: Enigma.

Rix, H. (2002). Sabellische Texte. Heidelberg: Winter.

Rix, H., \& Kümmel, M. (2001). Lexikon der indogermanischen Verben ( $2^{\text {nd }}$ ed.). Wiesbaden: Reichert.

Rozwadowski, J. (1948). Studia nad nazwami wód stowiańskich. Kraków: Polska Akademia Umiejętności.

Rudnicki, M. (Rev.). (1930). Compte rendu de: Vasmer, Beiträge zur alten Geographie der Gebiete zwischen Elbe und Weichsel (1929). Slavia Occidentalis, 9, 684-701.

Salskov Roberts, H. (1974). Five Tomb Groups in the Danish National Museum from Narce, Capena and Poggio Sommavilla. Acta Archaeologica, 45, 49-106.

Scheid, J. (1998). Commentarii Fratrum Arualium qui supersunt. Rome: École Française de Rome.

Schmalz, J. H. (1905). Kritische Kleinigkeiten. Berliner Philologische Wochenschrift, 18 März 1905, 366.

Schmid, W. P. (1989). Das Nehrungskurische, ein sprachhistorischer Überblick. In W. P. Schmid (Ed.), Nehrungskurisch (pp. 7-41). Mainz: Akademie der Wissenschaften und der Literatur / Wiesbaden - Stuttgart: Steiner.

Schmoll, U. (1959). Die Sprachen der vorkeltischen Indogermanen Hispaniens und das Keltiberische. Wiesbaden: Harrassowitz.

Schrijver, P. (1995). Studies in British Celtic Historical Phonology. Amsterdam - Atlanta: Rodopi.

Senart, É. (1934). Bṛhad-Āranyaka-Upaniṣad. Paris: Les Belles Lettres.

Seržant, I. A. (2014). The independent partitive genitive in Lithuanian. In A. Holvoet, \& N. Nau (Eds.), Grammatical Relations and their Non-Canonical Encoding in Baltic (pp. 257-299). Amsterdam - Philadelphia: John Benjamins.

Sievers, E. (1930). Zur Duenosinschrift. In Studies in Honor of Hermann Collitz (pp. 48-62). Baltimore: Johns Hopkins Press.

St. Marin, D. (1950). L'iscrizione di "Duenos". Atti della Accademia Nazionale dei Lincei, Memorie, Classe di Scienze morali, storiche e filologiche, Serie ottava, 2, 419-469.

Stefanelli, R. (2012). L'iscrizione di Duenos (CIL I ${ }^{2} 4$ ): Una proposta di lettura per la seconda sezione del testo. Archivio Glottologico Italiano, 97(2), 205-235.

Steinbauer, D. H. (1989). Etymologische Untersuchungen zu den bei Plautus belegten Verben der lateinischen ersten Konjugation. Altendorf b. Bamberg: Gräbner.

Strandberg, S. (2016). River Names. In C. Hough, \& D. Izdebska (Eds.), The Oxford Handbook of Names and Naming (pp. 104-114). Oxford: Oxford University Press.

Thurneysen, R. (1899). Inschriftliches. Zeitschrift für Vergleichende Sprachforschung, 35, 193-226.

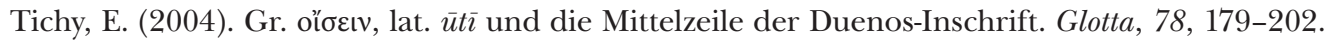


Tiffou, É. (1980). Remarques sur le vase de Duenos. In J.-B. Caron, M. Fortin, \& G. Maloney (Eds.), Mélanges d'études anciennes offerts à Maurice Lebel (pp. 223-230). St-Jean-Chrysostôme (Québec, Canada): Éditions du Sphinx.

Toporov [Топоров], В. Н. (1988). Балтийский элемент в гидронимии Поочья. І. Балто-славянские исследования, 1986, 154-177.

Trautmann, R. (1970). Baltisch-Slavisches Wörterbuch (2 ${ }^{\text {nd }}$ ed.). Göttingen: Vandenhoeck \& Ruprecht. Udolph, J. (1990). Die Stellung der Gewässernamen Polens innerhalb der alteuropäischen Hydronymie. Heidelberg: Winter.

Untermann, J. (1985). Los teónimos de la región lusitano-gallega como fuente de las lenguas indígenas. In J. de Hoz (Ed.), Actas del III coloquio sobre lenguas y culturas paleohispánicas (pp. 343-363). Salamanca: Ediciones Universidad de Salamanca.

Vanagas, A. (1981). Lietuviu hidronimu etimologinis žodynas. Vilnius: Mokslas.

Vasil'ev [Васильев], В. Л. (2009). Древнеевропейская гидронимия на Русском СевероЗападе. Балто-славянские исследования, 18, 262-278.

Vasmer, M. (1953-1958). Russisches etymologisches Wörterbuch. Heidelberg: Winter.

Vijūnas, A. (2008). Vedic nouns of the rétas- 'stream; semen' type and several related problems of Indo-European morphology. Acta Orientalia Vilnensia, 9(1), 7-15.

Villar, F. (1996). El Teónimo Lusitano Reve y sus Epítetos. In W. Meid, \& P. Anreiter (Eds.), Die grösseren altkeltischen Sprachdenkmäler (pp. 160-211). Innsbruck: Institut für Sprachwissenschaft der Universität Innsbruck.

Vincent, A. (1925). Les diminutifs de noms propres de cours d'eau particulièrement dans le domaine français. Revue belge de philologie et d'histoire, 4(1), 35-76.

Vine, B. (1999). A Note on the Duenos Inscription. In V. V. Ivanov, \& B. Vine (Eds.), UCLA Indo-European Studies 1 (pp. 293-305). Los Angeles: University of California.

Vottéro, G. (2002). Boeotica epigrammata. In J. Dion (Ed.), L'épigramme de l'Antiquité au XVIIe siècle ou du ciseau à la pointe (pp. 69-122). Nancy: ADRA.

Weiss, M. (2009). Outline of the Historical and Comparative Grammar of Latin. Ann Arbor - New York: Beech Stave Press.

Weiss, M. (2010). Language and Ritual in Sabellic Italy. Leiden - Boston: Brill.

Werba, C. H. (1997). Verba Indoarica. Pars I: Radices Primariae. Wien: Verlag der Österreichischen Akademie der Wissenschaften.

Willi, A. (2004). Flowing Riches: Greek ä $\varphi \varepsilon v o \varsigma$ and Indo-European Streams. In J. H. W. Penney (Ed.), Indo-European Perspectives. Studies in Honour of Anna Morpurgo Davies (pp. 323-337). Oxford: Oxford University Press.

Vincent Martzloff / martzloffvincent@gmail.com

Institute of Latin Studies

Paris - Sorbonne University

1, rue Victor Cousin, 75005 Paris, France 
Mgr. Barbora Machajdíková, Ph.D. / machajdikova.b@gmail.com

Department of Classical and Semitic Philology

Comenius University in Bratislava, Faculty of Arts

Gondova 2, 81499 Bratislava, Slovak Republic 
